\begin{tabular}{|c|c|}
\hline Title & A chirp-compensation technique using incident-angle changes of cavity mirrors in a femtosecond pulse laser \\
\hline Author(s) & Y amashita, Mikio; Torizuka, Kenji; Sato, Takuzo \\
\hline Citation & $\begin{array}{l}\text { IEEE Journal of Quantum Electronics, 23(11), 2005-2007 } \\
\text { https://doi.org/10.1109/JQE.1987.1073255 }\end{array}$ \\
\hline Issue Date & $1987-11$ \\
\hline Doc URL & http:/hdl.handle.net/2115/45321 \\
\hline Rights & $\begin{array}{l}\text { ( } 1987 \text { IEEE. Personal use of this material is permitted. However, permission to reprint/republish this material for } \\
\text { advertising or promotional purposes or for creating new collective works for resale or redistribution to servers or lists, } \\
\text { or to reuse any copyrighted component of this work in other works must be obtained from the IEEE. }\end{array}$ \\
\hline Type & article \\
\hline File Information & JQE23-11_2005-2007.pdf \\
\hline
\end{tabular}

Instructions for use 


\title{
A Chirp-Compensation Technique Using Incident- Angle Changes of Cavity Mirrors in a Femtosecond Pulse Laser
}

\author{
MIKIO YAMASHITA, KENJI TORIZUKA, AND TAKUZO SATO
}

\begin{abstract}
A technique for chirp-compensation in a CPM laser is presented. By using the change of the incident angle to multilayer dielectric cavity mirrors, the intracavity second-order dispersion $\ddot{\phi}(\omega)$ is adjusted without any additional elements. It is confirmed that the optimum value of $\ddot{\phi}(\omega)=+2.1 \times 10^{-28} \mathrm{~s}^{2}$ obtained when up-chirp was compensated and pulses as short as 55 fs were generated is reasonable, by comparison to analytic results of chirp behaviors. In addition, the effect of the third-order dispersion $\dddot{\phi}(\omega)$ at the optimum value of $\ddot{\phi}(\omega)$ on pulses is evaluated.
\end{abstract}

R ECENT studies on a colliding-pulse mode-locked (CPM) CW dye laser showed that the most important thing for femtosecond pulse generation is to compensate for the frequency chirp arising from dispersion and selfphase modulation in the cavity. For the chirp compensation, there are presently four techniques as follows: 1) the down-chirp compensation by the adjustment of the optical path in a prism inserted in the cavity [1], 2) the up-chirp compensation by the adjustment of the distance between four prisms inserted in the cavity [2], 3) the up-chirp compensation by the adjustment of the incident angle to a pair of Gires-Tournois interferometers used as parts of cavity mirrors [3], and 4) the up-chirp compensation by the use of the optimum second-order dispersion (the details will be described below) due to $\lambda_{0} / 4$ multilayer dielectric mirrors composing of the cavity [4]. The former three techniques necessitate the additional optical elements which furthermore complicate the optical alignment of the CPM ring laser. On the other hand, the last one has the inconvenience that cavity mirrors must be exchanged to other mirrors of different coatings to determine the optimum dispersion. In this paper we report that, by the change of the incident angle to cavity mirrors instead of the exchange of mirrors in the last technique, up-chirp can be compensated. In addition, it is shown that the amount of the second-order dispersion obtained for the up-chirp compensation agrees well with a recent analytic result of chirp behaviors due to intracavity self-phase modulation [5]. Furthermore, the influence of the third-order disper-

Manuscript received December 15, 1986; revised May 1, 1987. The authors are with the Laser Research Section, Radio- \& Optoelectronics Division, Electrotechnical Laboratory, Niihari-gun, Ibaraki-ken 305, Japan.

IEEE Log Number 8716535. sion due to mirrors at the optimum amount of the secondorder dispersion on femtosecond pulses is estimated.

The simple CPM (R6G + DODCI) laser used without additional elements is nearly identical to the one we previously described [4], except for the following points. The intracavity second-order dispersion was varied by moving mirrors $M_{6}$ and $M_{7}$ to the opposite direction of each other, as shown by arrow bars in Fig. 1(a). That is, the incident angles to mirrors $M_{6}$ and $M_{7}$, respectively, were varied from 45 to $60^{\circ}$ and from 40 to $25^{\circ}$. The details of the mirror coatings will be described below. The transmission of the output coupling mirrors $\left(M_{7}\right.$ or $\left.M_{1}\right)$ is about $0.5 \sim$ 1.0 percent around $630 \mathrm{~nm}$. Pump powers of $2.4-3.2 \mathrm{~W}$ at $514.5 \mathrm{~nm}$ were provided by a CW Ar ion laser. The DODCI concentration was $5 \times 10^{-3} \mathrm{M} / 1$ in a jet from a $39 \mu \mathrm{m}$ wide nozzle slit. Typical average output power was $10 \mathrm{~mW}$. The femtosecond pulse durations were measured by a background-free SHG autocorrelator $(0.2 \mathrm{~mm} \mathrm{KDP}$ crystal) operated in a fast-scan mode and a slow-scan mode. Hyperbolic secant-squared pulse shapes were assumed throughout in driving the pulse durations from the full width at half maximum of the intensity autocorrelation functions. The pulse spectra around $625-640 \mathrm{~nm}$ were monitored on an oscilloscope using an optical multichannel analyzer.

The phase of the EM wave of the laser pulse is shifted when the wave is reflected by a $\lambda_{0} / 4$ multilayer dielectric mirror. The phase shift $\phi(\omega)$ is a function of the optical angular frequency $\omega$ and can be calculated using a matrix formulation [6]. The effective phase-dispersion for chirp compensation is the second-order angular-frequency derivative $\ddot{\phi}(\omega)$ of the phase shift (the second-order dispersion). The quantity $\ddot{\phi}(\omega)$ is related to the group-velocity dispersion $\ddot{\kappa}(\omega)$ of a dispersive material with a length $l$ by an equation $\ddot{\phi}(\omega)=-l \ddot{\kappa}(\omega)(\kappa(\omega)$ : wavenumber $)$. Therefore, the positive second-order dispersion $\ddot{\phi}(\omega)$ corresponds to the negative group-velocity dispersion $\ddot{\kappa}(\omega)$. The value of $\ddot{\phi}(\omega)$ for the reflected laser pulse with a given angular frequency depends on the incident angle $\theta$ to a mirror and the resonance wavelength $\lambda_{0}$ of the multilayer stack of its mirror.

When the incident angle $\theta$ is larger than $45^{\circ}$ and the wavelength $\lambda_{d}$ of the incident laser-pulse is relatively longer than $\lambda_{0}$, the $\ddot{\phi}(\omega)$ has a large value $\left(10^{-29}-10^{-27}\right.$ 


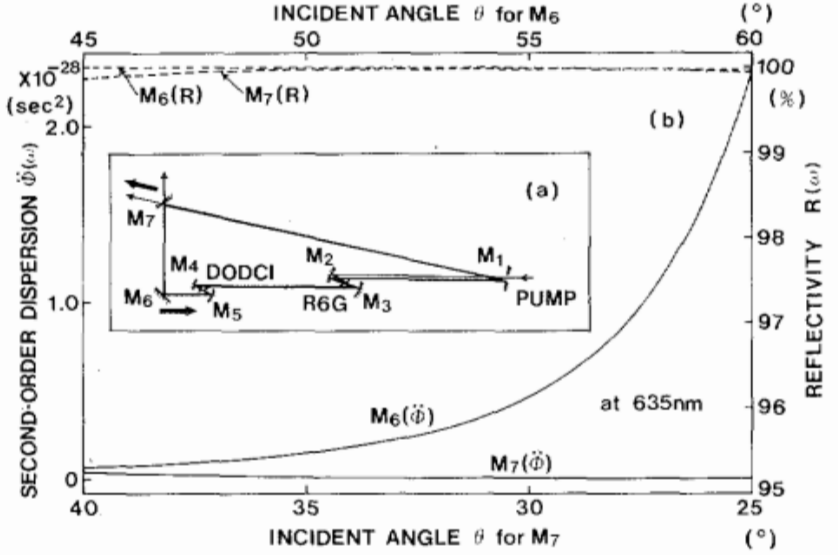

Fig. 1. (a) Cavity configuration of the simple CPM laser used. Two arrow bars represent the direction of the movement of mirrors $M_{6}$ and $M_{7}$ for the change of the incident angle $\theta$ of laser beams. (b) The second-order dispersion $\ddot{\phi}(\omega)$ and the reflectivity $R(\omega)$ at $635 \mathrm{~nm}$ plotted against the incident angle $\theta$ to mirrors $M_{6}\left(45\right.$ to $\left.60^{\circ}\right)$ and $M_{7}\left(40\right.$ to $\left.25^{\circ}\right)$.

$\mathrm{s}^{2}$ ) in the positive region and increases with the increase of the angle $\theta$. Our previous study showed that in a simple CPM laser up-chirp is dominant and compensated by a large value of $\ddot{\phi}(\omega) \simeq+1.8 \times 10^{-28} \mathrm{~s}^{2}$ in the positive region. Therefore, mirror $M_{6}$ where the angle $\theta$ is varied from 45 to $60^{\circ}$, should be made from two quarter-wave stacks to produce a increasingly positive value of $\ddot{\phi}(\omega)$ (around $10^{-28} \mathrm{~s}^{2}$ ) with keeping high reflectivity $R(\omega)$. That is, the resonance wavelength $\lambda_{0}$. up of the upper stack (the air side) for the incident angle $\theta=45-60^{\circ}$ should be relatively shorter than $\lambda_{d}$, so as to produce a value of $\ddot{\phi}(\omega)$ $=\sim 10^{-28} \mathrm{~s}^{2}$ in the positive region. The resonance wavelength $\lambda_{0} \cdot$ low of the lower stack (the substrate side) for the angle should be near $\lambda_{d}$, so as to keep high reflectivity. On the basis of these considerations, mirror $M_{6}$ was carefully made from two quarter-wave stack superimposed with $17 \lambda_{0}$. up $/ 4$ layers $\left(\lambda_{0} \cdot\right.$ up $\left.=563 \mathrm{~nm}\right)$ and $25 \lambda_{0}$. low $/ 4$ layers $\left(\lambda_{0}\right.$. low $\left.=653 \mathrm{~nm}\right)$ for $60^{\circ}$ incident angle. The coated layer materials of high and low refractive indexes were $\mathrm{TiO}_{2}\left(n_{H}=2.25\right)$ and $\mathrm{SiO}_{2}\left(n_{L}=1.46\right)$. Fig. 1(b) shows calculated dependences of the second-order dispersion $\ddot{\phi}(\omega)$ and reflectivity $R(\omega)$ on the incident angle $\theta$ (between 45 and $60^{\circ}$ ) to mirror $M_{6}$ for the $p$-polarization beam at $635 \mathrm{~nm}$.

As for mirror $M_{7}$, a mirror causing a small value of $\ddot{\phi}(\omega)$ around $\lambda_{d}$ is suitable, in order to keep its value almost constant at the incident angle between $\theta=40$ and $25^{\circ}$. Therefore, as the incident angle dependences of $\phi(\omega)$ and $R(\omega)$ are also shown in Fig. 1(b), a mirror which had been made from two quarter-wave stacks of 23 $\lambda_{0}$. up $/ 4$-layer $\left(\lambda_{0}\right.$ up $\left.=630 \mathrm{~nm}\right)$ and $24 \lambda_{0} \cdot$ low $/ 4$-layer $\left(\lambda_{0} \cdot\right.$ low $\left.=520 \mathrm{~nm}\right)$ for normal incidence, was used as mirror $M_{7}$ between $\theta=40$ and $25^{\circ}$.

For other mirrors $M_{1}-M_{5}$, the same mirrors as the previously employed ones [4] were used. Mirrors from $M_{2}$ to $M_{5}$ cause only a negligible small value of $\ddot{\phi}(\omega) \approx 5 \times$ $10^{-31} \mathrm{~s}^{2}$, while mirror $M_{1}$ causes a constant positive value of $\ddot{\phi}(\omega) \simeq+0.8 \times 10^{-28} \mathrm{~s}^{2}$ at about $630 \mathrm{~nm}$.
The effect on the pulse of the incident angle $\theta$ to cavity mirrors was experimentally examined by the simultaneous movement of mirrors $M_{6}$ and $M_{7}$ along the directions of the angle from 45 to $60^{\circ}$ and angle from 40 to $25^{\circ}$, [along arrow bars in Fig. 1(a)], respectively. At each $8 \mathrm{~mm}$ movement on translation stages, an optimum operating condition such as the pumping power and the alignment of the optical components was carefully adjusted, while monitoring the fast scanned autocorrelation traces on an oscilloscope. Consequently, the pulse duration dependence on the second-order dispersion $\ddot{\phi}(\omega)$ due to all cavity mirrors was obtained as shown in Fig. 2 . As the value of $\ddot{\phi}(\omega)$ increases from $0.8 \times 10^{-28}$ to $2.1 \times 10^{-28} \mathrm{~s}^{2}$, the pulse duration decreases from 90 to $55 \mathrm{fs}$, and then the further increase of $\ddot{\phi}(\omega)$ to $2.5 \times 10^{-28} \mathrm{~s}^{2}$ broadens the pulse duration to $68 \mathrm{fs}$. The obtained dependence and the value of $\ddot{\phi}(\omega)$ where the shortest pulses were generated, are in agreement with those in the previous experiment based on mirror exchange [4]. This indicates that the negative group-velocity dispersion corresponding to the above value of $\ddot{\phi}(\omega)$ can compensate for up-chirp mainly due to the effect of the nonlinear refractive index of the solvent of the DODCI absorber, ethylene glycol. We shall show below that the amount of this optimum dispersion is reasonable.

Miranda et al. carried out a simplified analysis of frequency-chirp $C(t)=\partial \delta \omega(t) / \partial t$ imposed on ferntosecond pulses interacting with a saturable absorber including the effect of nonlinear refractive index due to the solvent [5]. Their results showed that the chirp in a CPM laser remarkably depends on the intracavity pulse energy $E_{p}$. In the region of $E_{p} \leqslant 1 \mathrm{~nJ}$, down-chirp due to the transient saturation of the absorption is dominant around the pulse peak, while in the region of $E_{p} \geq 10 \mathrm{~nJ}$, up-chirp due to the time-dependent refractive index of the solvent is dominant. In the present CPM laser, $E_{p}$ is estimated to be $\sim 15$ $\mathrm{nJ}$, and, hence, up-chirp $C(0)>0$ at the pulse peak occurs. By using their numerical result for $C(0)$, which was obtained as a function of the pulse duration with a parameter $E_{p}, C(0)$ for the present CPM laser is estimated to be $\sim 10^{+26} \mathrm{rad} \cdot \mathrm{s}^{-2}$. Therefore, the amount of the second-order dispersion $\ddot{\phi}(\omega)$ necessary for this up-chirp compensation can be evaluated to be $\sim 10^{-28} \mathrm{~s}^{2}$ at the pulse duration of $T_{\text {in }}=55 \mathrm{fs}$ using an equation $\ddot{\phi}(\omega)=$ $C(0)^{-1} /\left[1+\left(8 \times(\ln 2)^{2} /\left(C(0) \times T_{\text {in }}^{2}\right)\right)^{2}\right]$ [7]. This agrees, in order of magnitude, with the experimental value.

Finally, we estimate the effect of the third-order angular-frequency derivative $\dddot{\phi}(\omega)$ of the phase shift (the third-order dispersion) due to mirrors on femtosecond pulses in the present CPM laser. The value of $\dddot{\phi}(\omega)$ due to all mirrors at the above obtained optimum value of $\ddot{\phi}(\omega)$ for up-chirp compensation is calculated to be $\sim 1$ $\times 10^{-41} \mathrm{~s}^{3}$. A few research groups derived an equation describing pulse broadening due to the third-order dispersion [8], [9]. Their results showed that for the input chirpcompensated pulse, the behavior of its output pulse shape is symmetric in respect to the sign of $\dddot{\phi}(\omega)$. As the value 


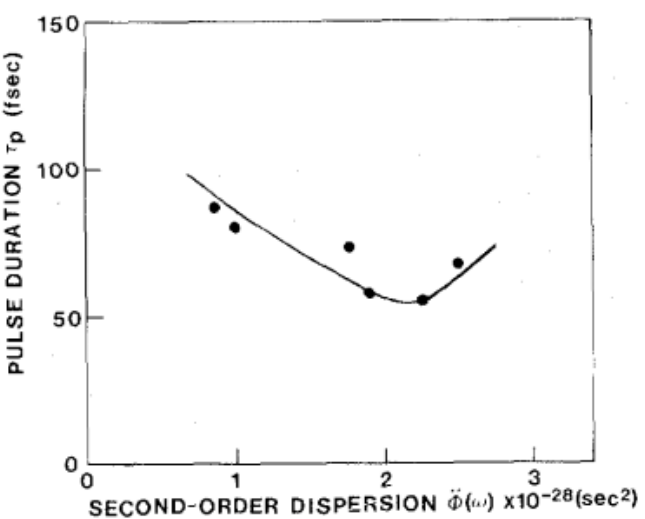

Fig. 2. Pulse duration as a function of the second-order dispersion $\ddot{\phi}(\omega)$ due to cavity mirrors, which was varied by the change of the incident angle to mirrors.

of $\dddot{\phi}(\omega)$ becomes large, the peak intensity of the main pulse decreases and some small pulses follow after the main pulse. From the application of their analysis to femtosecond pulses, it is found that when chirp-compensated $50 \mathrm{fs}$ pulses are reflected by mirrors of $\dddot{\phi}(\omega)=2 \times 10^{-41}$ $\mathrm{s}^{3}$, they are hardly broadened. However, when $30 \mathrm{fs}$ pulses are reflected by the same mirrors, they are broadened by about two times, and when being reflected by mirrors with less than $\dddot{\phi}(\omega)=4 \times 10^{-42} \mathrm{~s}^{3}$ they are hardly broadened. Therefore, for generation of pulses shorter than $50 \mathrm{fs}$, mirrors having $\dddot{\phi}(\omega) \leqslant 4 \times 10^{-42} \mathrm{~s}^{3}$ should be used.

In conclusion, it has been demonstrated as a technique of chirp-compensation in a CPM laser that the change of the incident angle to cavity mirrors has enabled us to adjust the intracavity second-order dispersion and, hence, to compensate for up-chirp and generate pulses as short as $55 \mathrm{fs}$. In addition, the evaluation on the effect of the thirdorder dispersion due to mirrors at the optimum secondorder dispersion has been done.

\section{REFERENCES}

[1] W. Dietel, J. J. Fontaine, and J.-C. Diels, "Intracavity puise compression with glass: A new method of generating pulses shorter than 60 fsec," Opt. Lett., vol. 8, pp. 4-6, Jan. 1983.

[2] J. A. Valdmanis, R. L. Fork, and J. P. Gordon, "Generation of optical pulses as short as 27 femtoseconds directly from a laser balancing selfphase modulation, group-velocity dispersion, saturable absorption, and saturation gain,"'Opt. Lett., vol. 10, pp. 131-133, Mar. 1985.

[3] J. Heppner and J. Kuhl, "Intracavity chirp compensation in a CPM laser using thin film interferometers," Appl. Phys. Lett., vol. 47, pp. 453-455, 1985 .

[4] M. Yamashita, M. Ishikawa, K. Torizuka, and T. Sato, "Femtosecond-pulse laser chirp compensated by cavity-mirror dispersion," Opt. Lett., vol. 11, pp. 504-506, Aug. 1986.

[5] R. S. Miranda, G. R. Jacobovitz, C. H. Brito Cruz, and M. A. F. Scarparo, "Positive and negative chirping of laser pulses shorter than
$100 \mathrm{fsec}$ in a saturable absorber," Opt. Lett., vol. 11, pp. 224-226, Apr. 1986.

[6] H. M. Liddell, Computer-Aided Techniques for the Design of Multilayer Filters. Bristol, England: Hilger, 1981.

[7] W. Dietel, E. Dopel, D. Kuhlke, and B. Wilhelmi, "Pulses in the femtosecond range from a $\mathrm{CW}$ dye ring laser in the colliding pulse mode-locking (CPM) regime with down-chirp," Opt. Commun., vol. 43, pp. 433-436, Nov. 1982.

[8] M. Miyagi and S. Nishida, "Pulse spreading in a single-mode fiber due to third-order dispersion," Appl. Opt., vol. 18, pp. 678-682, Mar. 1979.

[9] D. N. Christodoulides, E. Bourkoff, and R. J. Joseph, "Reflection of femtosecond optical pulses from multiple-layer dielectric mirrorsAnalysis," IEEE J. Quantum Electron., vol. QE-22, pp. 186-191, Jan. 1986.

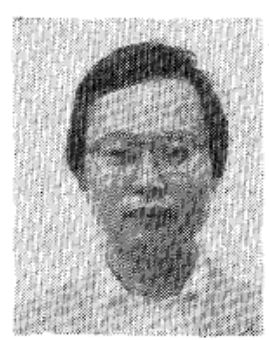

Mikio Yamashita was born in Kyoto, Japan, on February 15, 1946. He received the M.S. and $\mathrm{Ph} . \mathrm{D}$. degrees in electronics engineering from Kyoto University, Kyoto, Japan, in 1970 and 1976 , respectively.

Since joining the Laser Research Section, Radio- and Opto-Electronics Division, Electrotechnical Laboratory, Tokyo, Japan, in 1970, he has been engaged in research on laser excitation ESR studies on laser dyes, development of the tunable dye laser, and its application to the isotope separation. In 1978 he was a Visiting Research Associate at Imperial College. His research interests are currently the generation of the ultrashort pulse and its application including photochemical biotechnology.

Dr. Yamashita is a member of the Physical Society of Japan, the Japan Society of Applied Physics, and the Laser Society of Japan.

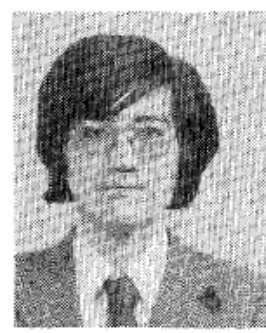

Kenji Torizuka was born in Saitama, Japan, on July 5,1957 . He received the M.S. and Ph.D. degrees in physics from the University of Tokyo, Tokyo, Japan, in 1982 and 1986, respectively.

Until 1985 he had been working on picosecond spectroscopy at the Institute for Solid State Physics, University of Tokyo, Tokyo, Japan. Since joining the Laser Research Section, Radio- and Opto-electronics Division, Electrotechnical Laboratory, Ibaraki, Japan, he has been working on femtosecond technology and application.

Dr. Torizuka is a member of the Physical Society of Japan and the Japan Society of Applied Physics.

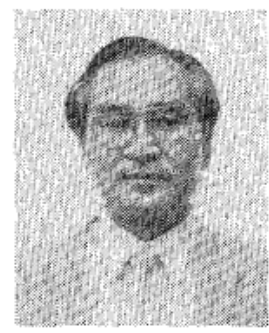

Takuzo Sato was born in Akita, Japan, on October 27,1940 . He received the M.S. and Ph.D. degrees in electronics engineering from Tohoku University, Sendai, Japan, in 1965 and 1980, respectively.

In 1965 he joined the Electrotechnical Laboratory, Tokyo, Japan, where he has been engaged in research on the $O$-switched ruby laser, dye lasers, mixing of laser beams, laser radar, and excimer lasers. His fields of interest are high-power lasers and their applications.

Dr. Sato is a member of the Institute of Electronics and Communication Engineers of Japan, the Japan Society of Applied Physics, and the Laser Society of Japan. 